includes $\mathrm{CD} 8+\mathrm{T}$ cells driving tumor regression. Our data demonstrate that ATRC-101, bound to its target which is an RNP complex, can activate myeloid cells and are consistent with this activation occurring via FcR and Toll-like receptor (TLR) pathways.

http://dx.doi.org/10.1136/jitc-2020-SITC2020.0689

\section{CD122-SELECTIVE IL-2 COMPLEXES TREAT OVARIAN CARCINOMAS, INDUCE TREG FRAGILITY AND PROMOTE T CELL STEM CELLS}

${ }^{1}$ Yilun Deng*, ${ }^{2} J u s t i n$ Drerup, ${ }^{1}$ Xinyue Zhang, ${ }^{1}$ Ryan Reyes, ${ }^{1}$ Jenny Mendez, ${ }^{1}$ Aravind Kancharla, 'Myrna Garcia, 'Haiyan Bai, 'Alvaro Padron, 'Harshita Gupta, ${ }^{1}$ Tyler Curiel. ${ }^{1}$ UT Health San Antonio, San Antonio, TX, USA; ${ }^{2}$ UT Southwestern, Dallas, TX, USA

Background Ovarian cancer (OC) responds poorly to immunotherapies. Regulatory $\mathrm{T}$ cells (Treg) engage IL-2 by high-affinity CD25 for differentiation and function, ${ }^{1}$ and anti-tumor effector $\mathrm{T}$ cells (Teff) use intermediate affinity CD122. We studied IL-2 complexes (IL-2c) that selectively activate CD122 (Teff) over CD25 (Tregs).

Methods Orthotopic ID8agg-luc mouse OC burden was measured by in vivo imaging. Tumor, ascites and draining lymph nodes (TDLN) were analyzed by flow and tSNE. IL-2c was complexed using $1.5 \mu \mathrm{g} /$ mouse IL-2 and $7.5 \mu \mathrm{g} /$ mouse aIL-2 (clone JES6-5H4) before i.p. injection every other day $\mathrm{x} 4$ starting at day 7. antiPD-L1 was given at 100ug/mouse every 3 days $\times 4$ starting from Day 11 . FIR mice ${ }^{2}$ were used to sort live Tregs.

Results IL-2c but not antiPD-L1 potently inhibits ID8agg (figure 1). IL-2c decreased ascites Treg functional markers (e.g., CD25, granzymeB) while upregulating the same markers on Teffs (figure 2). IL-2c inhibited Treg suppression in ascites while TDLN Tregs were unaffected (figure 3). tSNE showed great similarity of TDLN Tregs treated with isotype and IL-2c while ascites Tregs after IL-2c showed a fragile phenotype (e. g., increased PD-1, T-bet, and IFNgamma with maintained FoxP3 expression [figure 4]) which is known to contribute to better response to cancer immunotherapy. ${ }^{3} 4$ We observed a complete reduction of tumor bioluminescence with $\mathrm{IL}-2 \mathrm{c}$ and antiPD-L1 combo treatment in nearly all subjects significantly exceeding effect of IL-2c alone (figure 5). A CD8+CXCR5

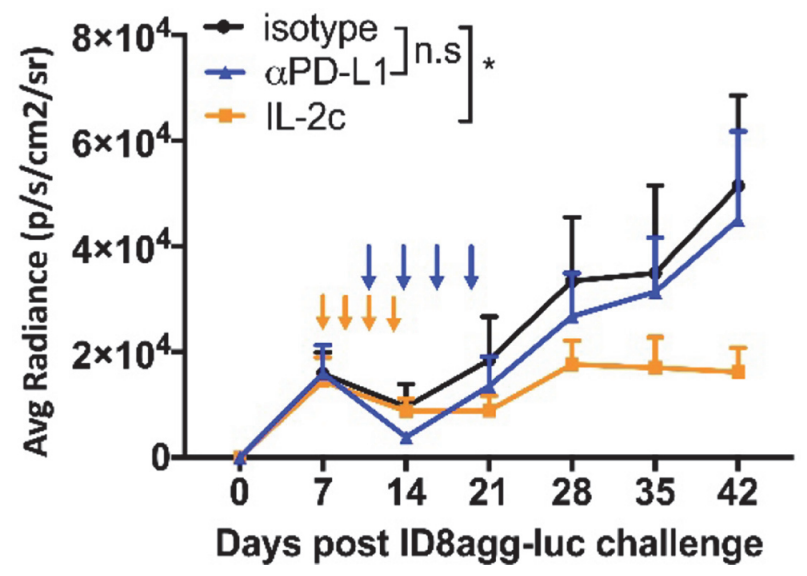

Abstract 690 Figure $1 \quad \mathrm{IL}-2 \mathrm{c}$ but not aPD-L1 treats ID8agg Luciferase signal of ID8agg-luc tumors treated with isotype, ?PD-L1, or $\mathrm{IL}-2 \mathrm{c}$ measured by in vivo imaging. Arrows indicate treatments.

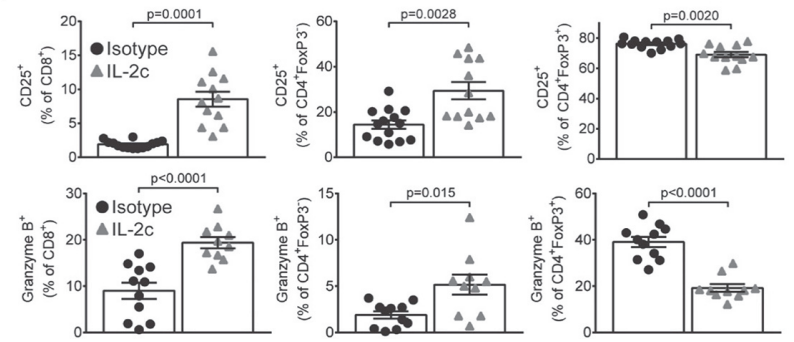

Abstract 690 Figure $2 \mathrm{IL}-2 \mathrm{c}$ inhibits functional markers on tregs and promotes teff

Expression of CD25 and granzymeB were measured by flow cytometry in indicated population from isotype or IL-2c treated ascites 3 weeks after final IL-2C dose.
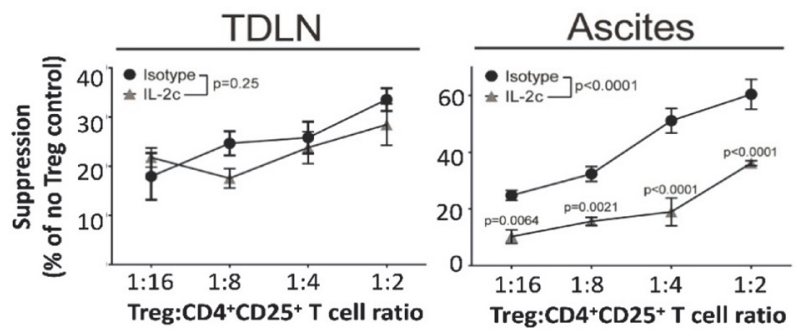

Abstract 690 Figure $3 \quad$ IL-2c reduces ascites Treg suppressive function

+TCF-1+ T cell stem cell (TCSC) population reportedly improves immune checkpoint blockade efficacy. ${ }^{5}{ }^{6}$ Since CD122 is regulated by TCF- $1,{ }^{7}$ we explored the effect of IL$2 \mathrm{c}$ on these TCSC. IL-2c significantly induced a CD8+TCF-1 + TCSC population in ID8agg tumors (figure 6), possibly through a positive feedback loop by further enhancing CD122 expression on TCF-1+, but not TCF-1- cells (figure 7). tSNE analysis of detailed immune phenotype of IL-2c induced TCSC revealed that these TCSC differed from those induced by antiPD-L1. In ID8agg, antiPD-L1-induced TCSC are mostly CXCR5 + and PD1+, consistent with previous reports in other cancers ${ }^{3}{ }^{4}$ while IL-2c-induced TCSC were PD1- (figure 8), expressed CCR2 and CXCR3, and produced TNFalpha (figure 9).

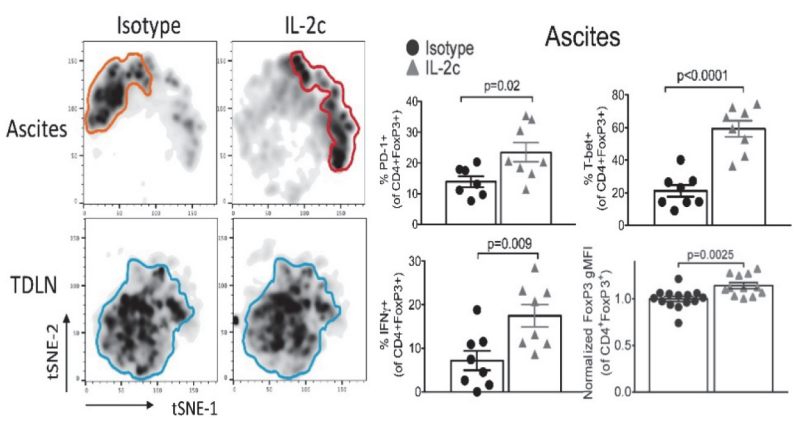

Abstract 690 Figure $4 \quad \mathrm{IL}-2 \mathrm{c}$ induces Treg fragility in ascites but not TDLN tSNE analysis on CD45+CD3+CD4+FoxP3+ cells from ascites and TDLN of isotype and IL-2c treated ID8agg-luc challenged mice. Right, representative bar graphs of flow data. 


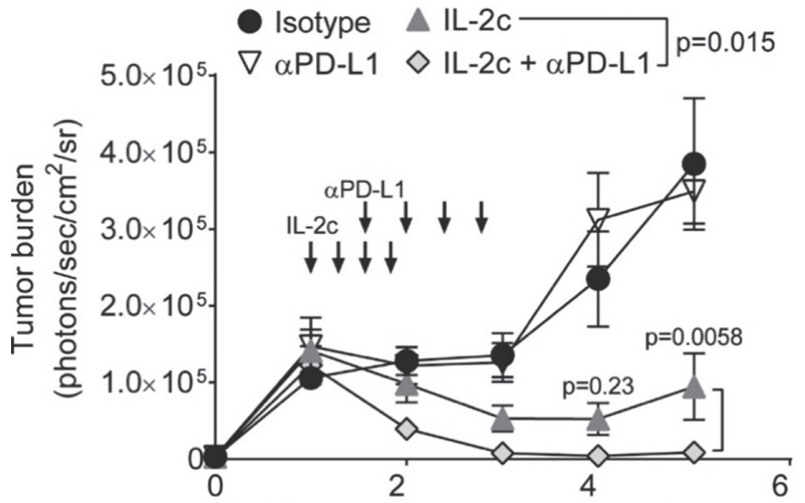

Abstract 690 Figure $5 \quad \mathrm{IL}-2 \mathrm{c}$ and aPD-L1 combo treatment effectively cures ID8agg

Luciferase signal of ID8agg-luc tumors treated with isotype, ?PD-L1, IL$2 \mathrm{c}$ or combo measured by in vivo imaging. Arrows indicate treatments.
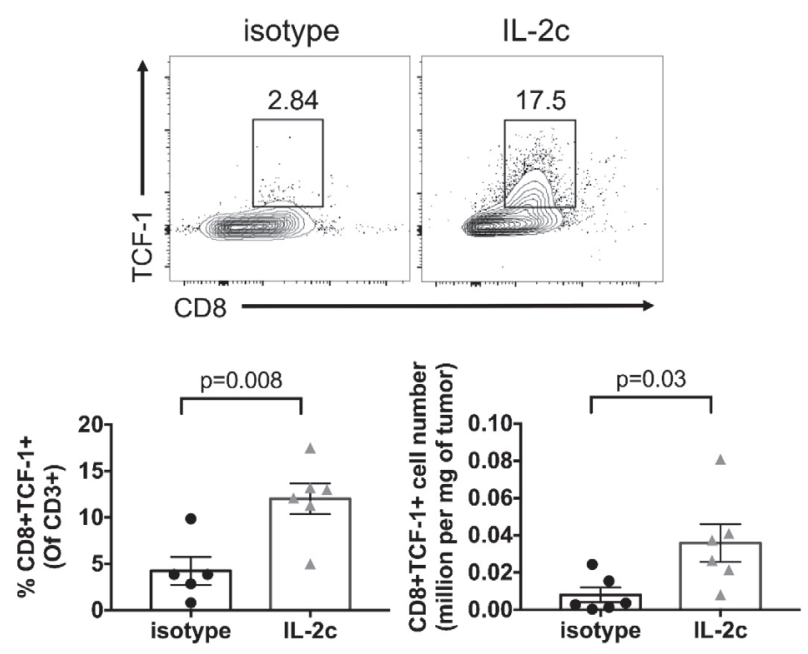

Abstract 690 Figure $6 \quad$ IL-2C induces a CD8+TCF-1+ population in ID8agg

ID8agg-luc tumors analyzed by flow cytometry gated on CD45+CD3 + CD8+ cells.

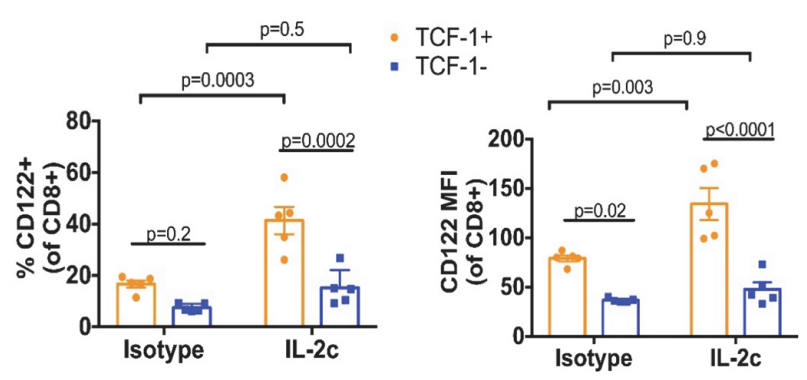

Abstract 690 Figure $7 \quad \mathrm{IL}-2 \mathrm{C}$ induces CD122 on CD8+TCF-1+ but not TCF-1 - cells

CD122 expression was measured by flow cytometry in CD8+TCF-1+ and CD8+ TCF-1- cells from tumors treated with isotype or IL-2C.

Conclusions We define two novel IL-2c effects: inducing Treg fragility therefore reducing immunosuppression while

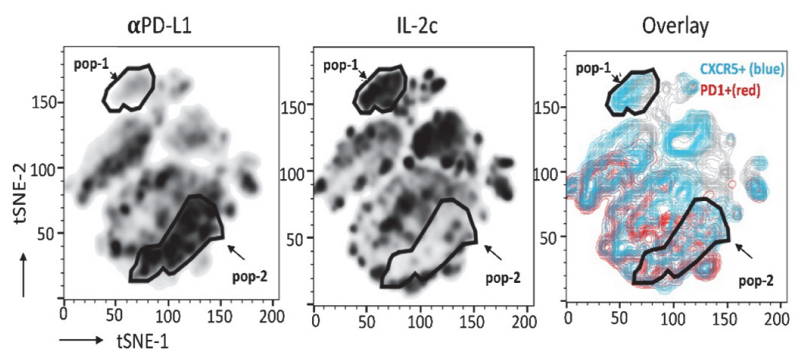

Abstract 690 Figure 8 CD8+TCF1+ cells from ID8agg analyzed by flow cytometry plus tSNE. Cells expressing CXCR5 (blue) and PD1 (red) indicated in overlay
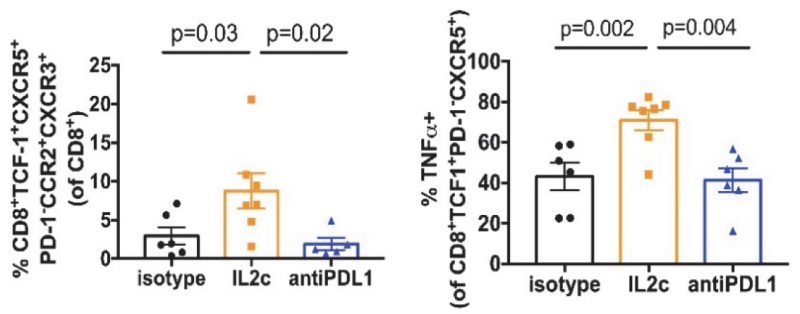

Abstract 690 Figure 9 CD8+TCF1+ cells from ID8agg analyzed by flow cytometry

promoting TCSC that could enhance effective anti-tumor immunity. Current work tests if effects are related and help efficacy, and mechanisms for IL-2c Treg effects. We also show that elicited TCSC differ by treatment and tumor, requiring additional investigations.

Acknowledgements This work is supported by CPRIT Research Training Award (RP 170345), Ovarian Cancer Research Alliance Ann and Sol Schreiber Mentored Investigator Award to YD and R01 CA205965to TC.

Ethics Approval All mice studies were approved by UT Health San Antonio Institutional Animal Care and Use Committee (IACUC). Approval number 20150093AR, 20140001AR, 20170035AR, 20140039AR, 20140027AR, 20090128AR, 20120071AR, 20180021AR.

\section{REFERENCES}

1. Malek TR: The biology of interleukin-2. Annu Rev Immunol 2008, 26:453-479.

2. Fantini MC, Dominitzki S, Rizzo A, Neurath MF, Becker C: In vitro generation of CD4+ CD25+ regulatory cells from murine naive T cells. Nat Protoc 2007, 2 (7):1789-1794.

3. Overacre-Delgoffe $A E$, Chikina $M$, Dadey RE, Yano $H$, Brunazzi EA, Shayan $G$, Horne W, Moskovitz JM, Kolls JK, Sander C, et al: Interferon-gamma Drives Treg Fragility to Promote Anti-tumor Immunity. Cell 2017, 169(6):1130-1141 e1111.

4. Overacre-Delgoffe AE, Vignali DAA: Treg Fragility: A Prerequisite for Effective Antitumor Immunity? Cancer Immunol Res 2018, 6(8):882-887.

5. Brummelman J, Mazza EMC, Alvisi G, Colombo FS, Grilli A, Mikulak J, Mavilio D, Alloisio M, Ferrari F, Lopci E, et al: High-dimensional single cell analysis identifies stem-like cytotoxic CD8(+) T cells infiltrating human tumors. J Exp Med 2018

6. Sade-Feldman M, Yizhak K, Bjorgaard SL, Ray JP, de Boer CG, Jenkins RW, Lieb DJ, Chen JH, Frederick DT, Barzily-Rokni M, et al: Defining T Cell States Associated with Response to Checkpoint Immunotherapy in Melanoma. Cell 2018, 175 (4):998-1013 e1020.

7. Jeevan-Raj B, Gehrig J, Charmoy M, Chennupati V, Grandclement C, Angelino P, Delorenzi M, Held W: The Transcription Factor Tcf1 Contributes to Normal NK Cell Development and Function by Limiting the Expression of Granzymes. Cell Rep 2017, 20(3):613-626.

http://dx.doi.org/10.1136/jitc-2020-SITC2020.0690 